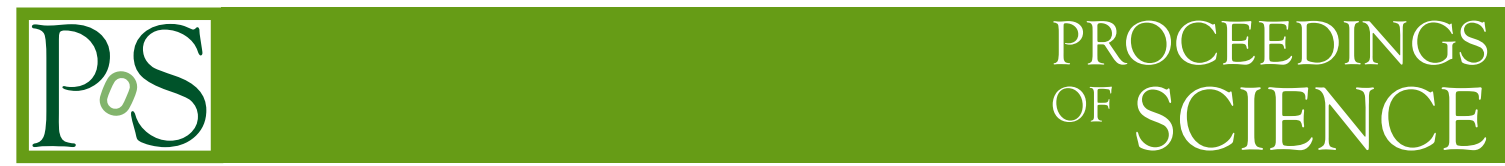

\title{
Intrinsic properties of the top quark
}

\section{Riccardo Di Sipio*, on behalf of the ATLAS Collaboration}

Università di Bologna

E-mail: disipioabo.infn.it

In this talk we review the most up-to-date results by the ATLAS Experiment at the LHC on the intrinsic properties of the top quark. By intrinsic we mean properties that define this particle uniquely, or that are a direct consequence of them. After a brief introduction, we will describe the measurements regarding the charge and the mass of the top quark, the polarization of $W$ bosons produced in top quark decays, and the search for flavor-changing neutral currents with top quarks.

XXII. International Workshop on Deep-Inelastic Scattering and Related Subjects, 28 April - 2 May 2014

Warsaw, Poland

* Speaker. 


\section{Introduction}

The top quark, first observed by the CDF experiments in 1995[1], was "rediscovered" at CERN by the ATLAS[2] and CMS experiments[3] and is now being studied with large data samples. The top quark is special among the particles because of its high mass. This fact prevents the formation of hadrons containing tops, so we have a unique opportunity to measure its properties directly before they are washed out by hadronization. Thanks to the high performance of the Large Hadron Collider (LHC) and of the two detectors during the Run $1(2011+2012)$ data taking, about six millions top quarks have been produced in total. Most of the measurements are already dominated by systematic uncertainties. It will be of great importance to pay a lot of effort into trying to understand and reduce these uncertainties, both of theoretical and experimental nature, before the start of the LHC data taking at $\sqrt{s}=13 \mathrm{TeV}$ that is scheduled for 2015 .

\section{Electric charge}

In the Standard Model, the top quark is an up-type quark so its charge is $+2 / 3$ of that of the proton. Shortly after the discovery of the top quark, an exotic alternative to the Standard Model was proposed[4] in which a top-like particle appears with charge $-4 / 3$. Measurements performed at Tevatron[5] strongly disfavored but did not exclude this other scenario. The analysis strategy adopted by ATLAS[6] relies on the fact that in the Standard Model the product of the charges of the lepton and that of the $b$-quark that come from top decays is always negative, while in the exotic model it is always positive. The sign of the top quark charge is transferred to the $W$ boson and then to the charged lepton, and it is quite easy to measure this quantity experimentally. This is not true for the $b$-quark, because the charge of a jet is not a well-defined quantity. Experimentally we make use of a weighted sum of the track charges as proxy for this quantity.

A harsh set of cuts is applied to the collected events to isolate a very pure sample. The observable is then compared against the two models. The resulting top charge is $0.64 \pm 0.02($ stat $) \pm$ 0.08 (syst), compatible with $+2 / 3$, as predicted by the Standard Model. The exotic model is now excluded by more than 8 standard deviations. We conclude that the observed particle is compatible to be an up-type quark.

\section{Mass}

The most defining property is probably the mass of a particle. In the case of the top quark, its mass plays an important role in particle physics, mainly because of its large coupling to the Higgs boson. Calculations at next-to-next to leading order, assuming the Standard Model to be valid up tho the highest energies, suggest that the Higgs potential might be unstable at high energies close to the GUT scale $\left(10^{16} \mathrm{GeV}\right)$, with this feature strongly dependent on the value of the top pole mass[7]. Setting aside the theoretical implications, from an experimental perspective this gives a strong motivation for a precise determination of this parameter.

Usually, the top mass is measured as an invariant mass of its decay products, with corrections applied using Monte Carlo simulations. Unfortunately, beyond the leading order its value depends on the renormalization scheme, which is not well-defined in current Monte Carlo generators. This 
leaves us with an ambiguous definition when the uncertainty is below $1 \mathrm{GeV}$ and close to the QCD scale $\left(\Lambda_{Q C D} \sim 0.5 \mathrm{GeV}\right)$.

ATLAS performed several measurements of the top mass. The most precise one[8] is obtained with a three-dimensional fit to constrain the largest systematics. The first step is the full reconstruction of the $t \bar{t}$ decay kinematics on an event-by-event basis. This is accomplished in the lepton+jets channel with a maximum likelihood approach, whose inputs are the four jets, the charged lepton and the missing transverse energy. The kinematic fit extracts the top mass using the jets permutation with the highest likelihood. This information is used to reconstruct the mass of the hadronicallydecaying $W$ boson, which depends on the jet energy scale, and a parameter called $R_{l b}$ defined as the ratio between the transverse momenta of $b$-jets and light-jets in the hadronic decay of the top quark. This quantity, being a ratio, is independent of the jet energy scale by construction. A large number of templates are generated for different top mass hypotheses in the experimentally allowed range, and for different energy-scale factors of light jets (JSF) and $b$-jets (bJSF). An unbinned 3-dimensional fit extracts the most probable values of the top mass and the energy scale factors. These factors turn out to be close to unity. The total uncertainty on the final result is less than $1 \%$ (fig. 1).

$$
\begin{aligned}
m_{t} & =172.31 \pm 0.75(\text { stat }+J S F+b J S F) \pm 1.35(\text { syst }) \mathrm{GeV} \\
J S F & =1.014 \pm 0.003(\text { stat }) \pm 0.021(\text { syst }) \\
b J S F & =1.006 \pm 0.008(\text { stat }) \pm 0.020(\text { syst })
\end{aligned}
$$

The main uncertainties are the $b$-tagging efficiency, the jet energy scale and the statistical component of the $b$-jet energy scale, which will be reduced with more data. To better understand the result and to compare it directly with the previous measurement, this procedure was applied also by fixing the $b$-jet energy scale factor to unity. This reduces the statistical uncertainty, but the main systematics are less constrained. The results obtained with the two methods agree within about 0.5 $\mathrm{GeV}$, which is less than the largest systematic uncertainty. The improvement with respect to the previous measurement is due to the reduction of a number of systematics, in particular the $b$-jet energy scale, the signal simulation modeling, the method to reconstruct the decay kinematics and the analysis strategy with the 3-dimensional fit.

It is also possibile to extract the value of the top mass in di-leptonic decays[9] thanks to the fact that the shape of the invariant mass distribution of the lepton and the $b$-jet $\left(m_{l b}\right)$ depends on the mass of the parent particle. In events with more than one $b$-jet, the combination with the lowest $m_{l b}$ is chosen. We compared the data against a set of $m_{l b}$ templates for different values of the top mass. The extracted best-fit value is compatible with the most precise measurement, and was also used in the combination of the ATLAS top mass average. The largest uncertainties are due to statistics, jet and $b$-jet energy scale, $b$-tagging efficiency.

\section{Polarization of $W$ bosons}

The structure of the interaction that is responsible for the decay of the top quark determines exactly whether the $W$ bosons from the top-quark decay are produced along preferred directions. In the Standard Model it is the $V-A$ electroweak vertex that fixes the angular distribution of the decay 


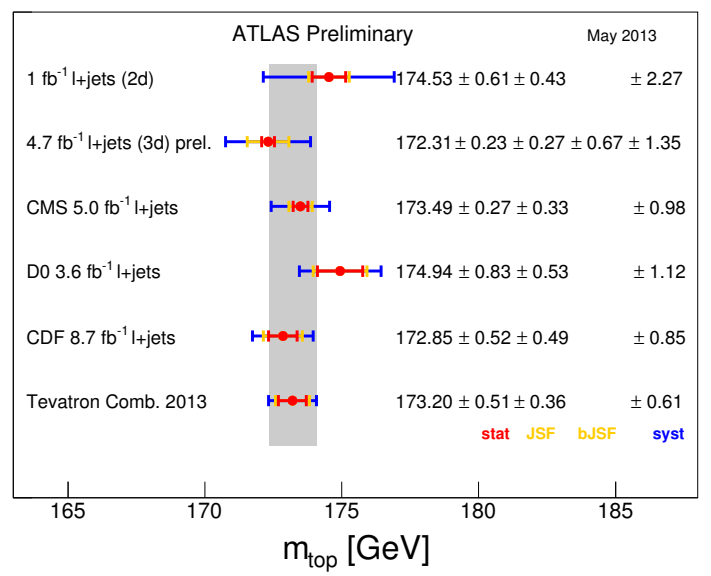

Figure 1: Compilation of measurements of the top-quark mass from different experiments.

products. This expression depends on three parameters, called helicity fractions, that have been calculated at NNLO precision[10]. If the interaction is non-standard, the angular distributions are modified. This can be parametrized in the context of Effective Field Theories, that add additional couplings to the usual ones. The role of the experiments is to set limits on these effective couplings, under some assumptions. In the Standard Model they are exactly zero.

Making use of the high correlation between the direction of flight of the top quark and the charged lepton produced in its decay, the helicity fractions can be extracted in two ways: one is to compare the angle between the $b$-quark and the charged lepton in the top rest frame $\left(\cos \theta^{*}\right)$ against a set of templates generated with different hypotheses on the value of the helicity fractions. Similarly, the $\cos \theta^{*}$ distribution can be translated in the so-called angular asymmetry. Putting all together, ATLAS extracted the best-fit values of the helicity fractions[11], that turn out to be compatible with the Standard Model expectations (fig. 2):

$$
\begin{aligned}
& F_{0}=0.67 \pm 0.03(\text { stat }) \pm 0.06(\text { syst }) \\
& F_{L}=0.32 \pm 0.02(\text { stat }) \pm 0.03(\text { syst }) \\
& F_{R}=0.01 \pm 0.01(\text { stat }) \pm 0.04(\text { syst })
\end{aligned}
$$

From these values, limits are set on the effective couplings $g_{L}$ and $g_{R}$, which are compatible with zero. Recently, the same measurements made by ATLAS and CMS were combined to give more precise measurements[12]. The two measurements are compatible, so the same conclusions hold true.

\section{Flavor-Changing Neutral Currents}

In the Standard Model, the top quark decays to a $W$ boson and a $b$-quark with a branching ratio very close to unity. Non-standard couplings can affect the way a top quark can be produced or decays, as for example $t g q, t Z q$ or $t \gamma q$ couplings. In the Standard Model, these couplings do not exist at tree level, and are GIM-suppressed at higher orders. 

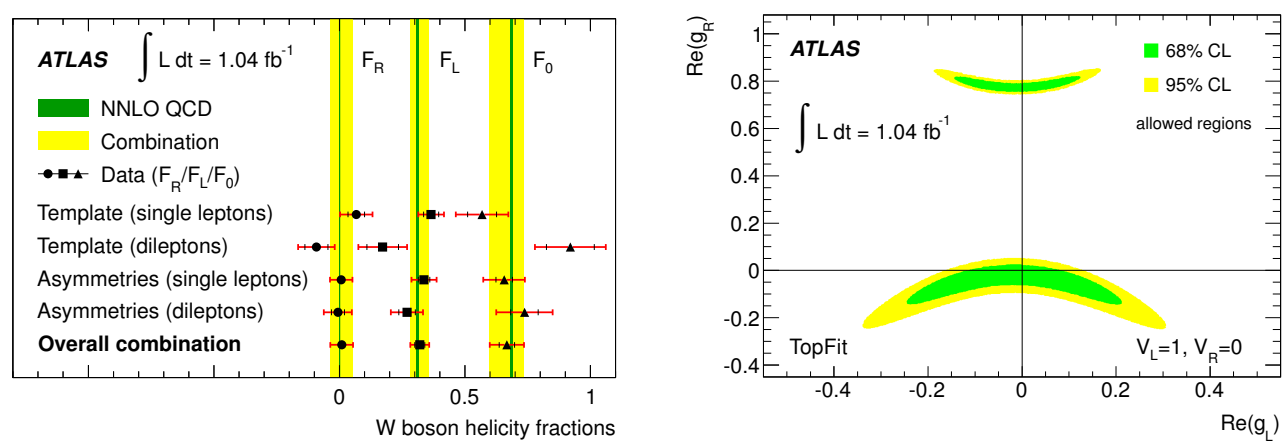

Figure 2: Helicity fractions (left) and effective theory couplings (right) measured by ATLAS.

The tgq coupling is studied looking for deviations in single top production rate[14]. In this case, their decays are assumed to be standard, but they are produced via a flavor-changing quarkgluon interactions $(q g \rightarrow t)$. A neural network approach extracts limits on the anomalous production rate.

In a second search, non-standard top decays are searched[13] in events with three charged leptons, assuming a standard top-antitop production mechanism. In this case, one of the tops decays to a $b$-quark and a $\mathrm{Z}$ boson, which in turn decays to two charged leptons (fig. $3 \mathrm{~b}$ ).

In both cases, the results are in good agreement with the Standard Model, and only limits are set (fig. 3a).

$$
\begin{aligned}
\sigma(q g \rightarrow t) \cdot B R(t \rightarrow b W) & <2.5 \mathrm{pb} @ 95 \% \mathrm{CL} \\
B R(t \rightarrow u g) & <3.1 \cdot 10^{-5} k_{\text {ugt }} / \Lambda<5.1 \cdot 10^{-3} \mathrm{TeV}^{-1} \\
B R(t \rightarrow c g) & <1.6 \cdot 10^{-4} k_{c g t} / \Lambda<1.1 \cdot 10^{-2} \mathrm{TeV}^{-1} \\
B R(t \rightarrow Z q) & <0.73 \% @ 95 \% \mathrm{CL}
\end{aligned}
$$

\section{Conclusions}

Thanks to the outstanding performance of the LHC, the experimental physics of the top quark entered the precision era, with most measurements already dominated by systematic uncertainties. The mass of the top quark is now known with a precision below $1 \mathrm{GeV}$, with the uncertainties that will become smaller and smaller with more data. The top quark is a unique probe to look for phenomena beyond the Standard Model, however the searches carried out with Run 1 data did not show any significant deviation from the Standard Model predictions.

\section{References}

[1] Observation of Top Quark Production in $\bar{p} p$ Collisions with the Collider Detector at Fermilab, The CDF Collaboration, Phys. Rev. Lett. 74(1995)2626.

[2] The ATLAS Experiment at the CERN Large Hadron Collider, ATLAS Collaboration, JINST 3 (2008) S08003. 


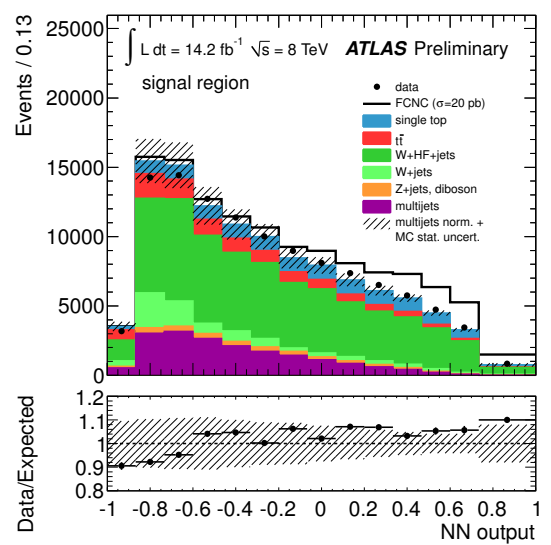

(a) FCNC in production of single tops.

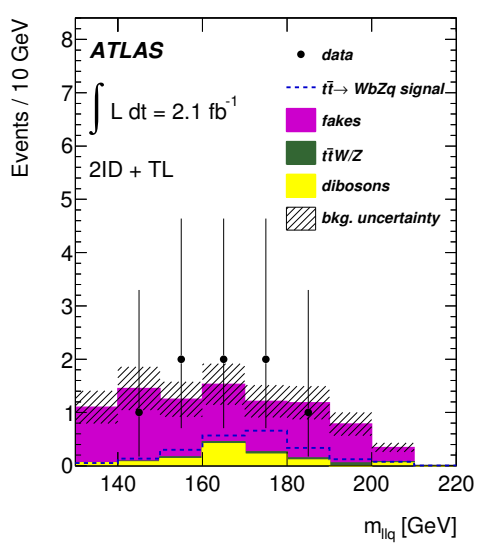

(b) FCNC in decay in $t \bar{t}$ event.

Figure 3: Output of the Neural Network in the search for FCNC in single top events (left) and invariant mass of $l l q$ to search for $t \rightarrow Z q$ anomalous decays .

[3] The CMS experiment at the CERN Large Hadron Collider, The CMS Collaboration, 2008 JINST 3 S08004.

[4] Alternative interpretation of the Fermilab Tevatron top events, D. Chang et al., Phys. Rev. D 59, 091503(R)

[5] Exclusion of an Exotic Top Quark with -4/3 Electric Charge Using Soft Lepton Tagging, T. Aaltonen et al. (CDF Collaboration), Phys. Rev. Lett. 105, 101801

[6] Measurement of the top quark charge in pp collisions at $\sqrt{s}=7 \mathrm{TeV}$ with the ATLAS detector, ATLAS Collaboration, JHEP11(2013)031

[7] Higgs mass implications on the stability of the electroweak vacuum, J. Elias-Miro et al., Phys.Lett. B709 (2012) 222-228

[8] Measurement of the Top Quark Mass from $\sqrt{s}=7 \mathrm{TeV}$ ATLAS Data using a 3-dimensional Template Fit, ATLAS Collaboration, ATLAS-CONF-2013-046, https://cds.cern.ch/record/1547327

[9] Measurement of the Top Quark Mass in Dileptonic Top Quark Pair Decays with $\sqrt{s}=7$ TeV ATLAS Data, ATLAS Collaboration, ATLAS-CONF-2013-077, https://cds.cern.ch/record/1562935

[10] Spin determination of single-produced resonances at hadron colliders, Y. Gao et al., Phys.Rev.D 81(2010)

[11] Measurement of $W$ boson polarization in top quark decays with the ATLAS detector, ATLAS Collaboration, JHEP 1206(2012)088

[12] TOPLHCWG: Combination of the ATLAS and CMS measurements of $W$ polarisation in top events (Autumn 2012), TOPLHCWG, ATLAS-CONF-2013-033, https://cds.cern.ch/record/1527531 and CMS-PAS-TOP-12-025, https://cds.cern.ch/record/1528567

[13] A search for flavour changing neutral currents in top-quark decays in pp collision data collected with the ATLAS detector at $\sqrt{s}=7 \mathrm{TeV}$, ATLAS Collaboration, JHEP 09(2012)139

[14] Search for single top-quark production via FCNC in strong interactions in $\sqrt{s}=8 \mathrm{TeV}$ ATLAS data, ATLAS Collaboration, ATLAS-CONF-2013-063, https://cds.cern.ch/record/1562777 